\title{
The AGILE contribution to GRBs studies
}



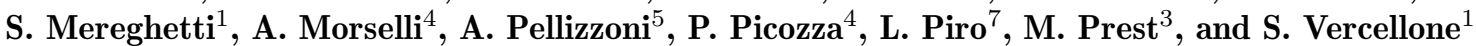 \\ 1 Istituto di Fisica Cosmica "G. Occhialini", CNR, Milano, Italy \\ 2 Columbia University, New York, U.S.A. \\ 3 University of Trieste and INFN, Trieste, Italy \\ 4 University of Roma "Tor Vergata" and INFN, Roma, Italy \\ 5 Agenzia Spaziale Italiana, Roma, Italy \\ 6 Istituto di Tecnologie e Studio delle Radiazioni Extraterrestri, CNR, Bologna, Italy \\ 7 Istituto di Astrofisica Spaziale, CNR, Roma, Italy
}

Received January 21; accepted May 28, 1999

\begin{abstract}
AGILE is a gamma-ray mission planned to be operating as an Observatory during the period 2002-2005. Its baseline instrument is designed to detect gamma rays in the $30 \mathrm{MeV}-50 \mathrm{GeV}$ band. AGILE's good sensitivity, very large field of view ( $\sim 1 / 5$ of the whole sky), and excellent timing capability $(\sim 1 \mathrm{~ms}$ deadtime) are ideal to study gamma-ray bursts (GRBs). AGILE is expected to detect $\sim 10$ GRBs per year at energies above $100 \mathrm{MeV}$. The Super-AGILE option might be able to localize GRBs within a few arcminutes and provide additional information in the hard X-ray band. A rapid alert program is an essential part of the scientific goals of the AGILE Observatory.
\end{abstract}

Key words: gamma-ray: bursts

\section{Introduction}

The gamma-ray mission AGILE (Astro-rivelatore Gamma a Immagini LEggero) was proposed in 1997 to the Italian Space Agency (ASI) Program of Small Scientific Missions (Tavani et al. 1999a). AGILE is a light and cost-effective mission ( $60 \mathrm{~kg}$ instrument, $\leq 200 \mathrm{~kg}$ in total) optimized for gamma-ray imaging and fast timing detection. Gamma-ray imaging in the energy range $30 \mathrm{MeV}-$ $50 \mathrm{GeV}$ is based on silicon tracking detectors developed for space missions by INFN and Italian University laboratories during the past years (Barbiellini et al. 1995; Morselli et al. 1995). AGILE's field of view (FOV) is unprecedently large $(\geq 1 / 5$ of the whole sky) because of state-of-the-art readout electronics and segmented anticoincidence system. The baseline instrument is designed

$\overline{\text { Send offprint requests to }}$ F. Longo: longof@ts.infn.it to achieve an on-axis sensitivity comparable to that of EGRET on board of CGRO (a smaller background resulting from an improved angular resolution more than compensates the loss due to a smaller effective area) and a better sensitivity for large off-axis angles (up to $\sim 60^{\circ}$ ). Planned to be operational during the year 2002 for a 3year mission, AGILE will ideally "fill the gap" between EGRET and GLAST.

Figure 1 shows a lateral view of the baseline instrument. Spectral information $(\Delta E / E \sim 1)$ is obtained by multiple scattering of created pairs in tungsten-silicon planes (for energies less than $\sim 500 \mathrm{MeV}$ ) and by the use of a mini-calorimeter (e.g., Morselli et al. 1999). The Super-AGILE option ${ }^{1}$ is based on an additional ultra-light coded-mask imaging system positioned on top of the baseline instrument. An additional silicon plane detector is planned to be sensitive in the energy band $\sim 10-40 \mathrm{keV}$. The imaging capability of Super-AGILE is designed to provide $\sim 1-2$ arcmin positions for GRBs.

\section{GRBs studies with AGILE}

GRBs can emit a substantial fraction of their energy above $100 \mathrm{MeV}$ both during their prompt emission phase and during the afterglow. About 10 GRBs have been detected by EGRET's spark chamber during $\sim 7$ years of operations (Dingus 1995; Schneid et al. 1996). This number appears to be limited by the EGRET FOV and sensitivity and not by the GRB emission mechanism. The GRB detection rate by AGILE is expected to be a factor of $\gtrsim 5$ larger than that of EGRET, i.e., $\geq 5-10$ events/year. We note that AGILE's flux sensitivity for GRB detection is comparable to that of EGRET that typically detected

\footnotetext{
1 Super-AGILE is an innovative concept currently developed by the AGILE Team with E. Costa and collaborators.
} 


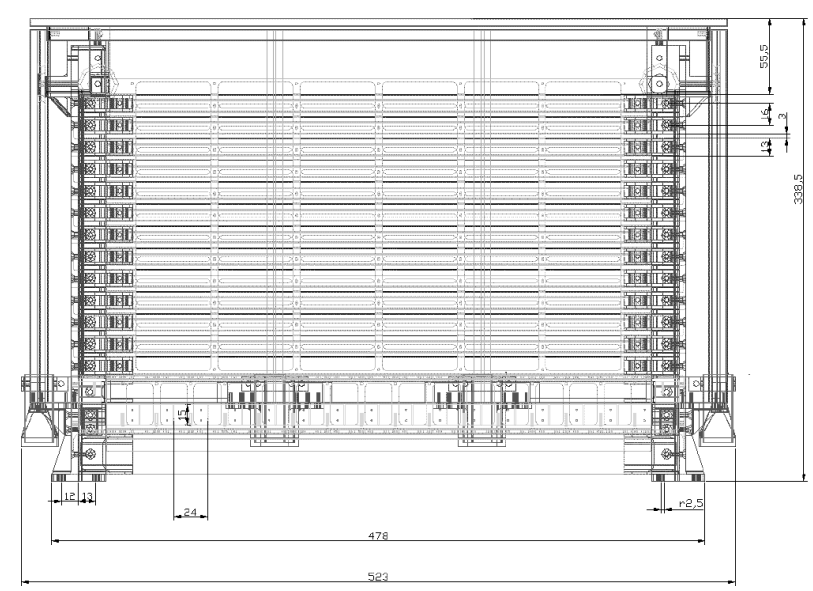

Fig. 1. Lateral cross-section of the AGILE baseline instrument. The anticoincidence system of plastic scintillator panels surrounds the detector made of $10 \mathrm{~W}$-Si planes $\left(0.7 X_{\mathrm{o}}\right)$ plus 2 more Si-only planes and a CsI mini-calorimeter $\left(1.5 X_{\mathrm{o}}\right)$. The baseline payload size is $\sim 53 \times 53 \times 35 \mathrm{~cm}^{3}$, and $\sim 53 \times 53 \times 44 \mathrm{~cm}^{3}$ for Super-AGILE

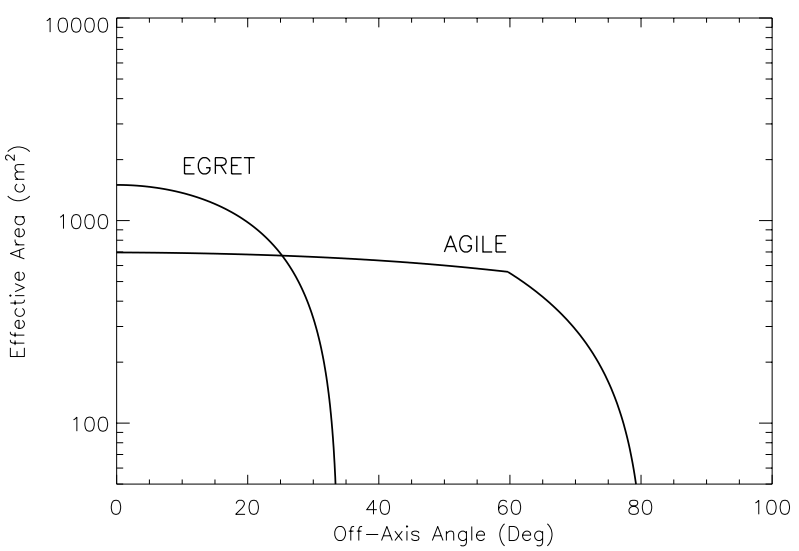

Fig. 2. Effective area at $1 \mathrm{GeV}$ as a function of photon incidence angle for AGILE (Tavani et al. 1999b) and EGRET (Thompson et al. 1993)

GRBs off-axis. Figure 2 shows the effective areas of AGILE and EGRET.

A short deadtime for gamma-ray detection $(\sim 1 \mathrm{~ms}$, i.e., a factor of $\sim 100$ times smaller than that of EGRET's spark chamber) is obtained by AGILE's fast electronic readout and a cyclic buffer. GRB pulses during the typically short risetimes $(\lesssim 0.5 \mathrm{~s})$ can be efficiently studied for the first time. Furthermore, AGILE might discover an hypothetical and theoretically expected class of events, i.e., high-energy very short GRBs $(\sim 0.1-1$ s $)$ that could not be efficiently detected above $100 \mathrm{MeV}$ with previous instrumentation.

Long events that we could call "gamma-ray afterglows" (lasting $\sim 100-1000 \mathrm{~s}$ ) can also be detected. The remarkable discovery by EGRET of gamma-ray emission up to $\sim 20 \mathrm{GeV}$ for GRB 940217 (Hurley et al. 1994) lasting $\sim 5,400$ s shows that gamma-rays can be emitted during a timescale much longer than that observed in the hard $\mathrm{X}$-ray range. Obviously, this fact suggests the existence of a quasi-continuous acceleration process shifting the peak of the spectral power per energy decade at photon energies substantially larger than those typical of the prompt pulse emission. This late-time particle acceleration challenges theoretical models for the early GRB afterglow emission. AGILE can contribute in obtaining more information on these enigmatic events. Currently, 2 out of 10 events detected by EGRET, GRB 930131 (Sommer et al. 1994) and GRB 940217 (Hurley et al. 1994) clearly show durations (in the gamma-ray band above $\sim 30 \mathrm{MeV}$ ) longer by a factor of $\sim 10$ or more than the durations established in the $50-300 \mathrm{keV}$ by BATSE (Paciesas et al. 1998).

AGILE is also expected to be quite efficient in detecting photons above $1 \mathrm{GeV}$ because of limited backscattering from a mini-calorimeter of small radiation length. For relatively bright events, GRB error boxes derived by gamma-ray data alone are expected to have radii smaller by a factor of $\sim 2$ than those of EGRET.

GRB broad-band spectral data from $\sim 1 \mathrm{MeV}$ up to $\sim 10 \mathrm{GeV}$ can be obtained by the baseline instrument by combining information of the CsI mini-calorimeter and silicon tracker. Additional information at lower energies can be obtained by the response of silicon tracker planes to the passage of hard X-rays differentially absorbed or Compton scattered throughout the tracker.

The Super-AGILE ultra-light coded mask system is planned to provide additional spectral information in the $\sim 10-40 \mathrm{keV}$ range. A relatively unambiguous "trigger" for a fast search in tracker data of GRB-related events can be obtained by ratemeter information from the additional silicon plane with readout electronics optimized in that energy range.

Super-AGILE will be able to locate GRBs within a few arcminutes and will systematically study the interplay between hard X-ray and gamma-ray emissions. A rapid alert and communication of quicklook analysis results is planned in case of GRB detection by Super-AGILE.

\section{References}

Barbiellini G., et al., 1995, NIM. A 354, 547

Dingus B.L., 1995, Astrophys. Space Sci. 231, 187

Hurley K., et al., 1994, Nat 372, 652

Morselli A., et al., 1995, in XIV Int. Cosmic Ray Conf. 3, 669

Morselli A., et al., 1999 (to appear in Mem. Soc. Astr. Ital.) astro-ph/9901183

Paciesas W.S., et al., 1998, http://gammaray.msfc.nasa.gov/ batse/data/grb/4b

Schneid E.J., et al., 1996, AIP Conf. Proc. 384, 253

Sommer M., et al., 1994, ApJ 422, L63

Tavani M., et al., 1999a (to appear in Mem. Soc. Astr. Ital.) astro-ph/9812096

Tavani M., et al., 1999b, The AGILE Mission, http://www.ifctr.mi.cnr.it/Agile

Thompson D.J., et al., 1993, ApJS 86, 629 